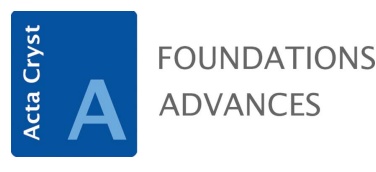

ISSN 2053-2733

Received 22 February 2017

Accepted 28 February 2017

Keywords: bicrystallography; coincidence site lattice; interfaces.

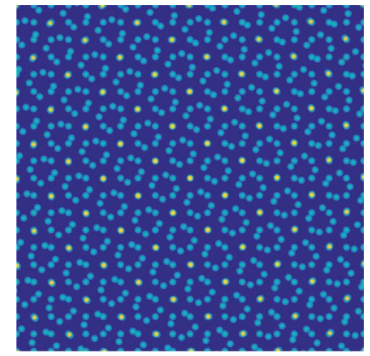

C 2017 International Union of Crystallography

\section{Approximating coincidence - turning a new page for bicrystallography}

\author{
Srikanth Patala* \\ Department of Materials Science \& Engineering, North Carolina State University, Raleigh, NC 27695, USA. \\ *Correspondence e-mail: spatala@ncsu.edu
}

Most materials used in engineering applications are polycrystalline, i.e. they consist of many single-crystal grains that come together to form bulk polycrystalline microstructures. In these polycrystals, the least understood defect types are the interfaces, which are present at the intersections of abutting grains with distinct lattice orientations. Since the individual grains have an underlying lattice structure and correspond to specific space-group symmetries, the properties of interfaces are analysed by first decoding the crystallographic aspects of the two overlapping crystals (i.e. a bicrystal). This framework, known as bicrystallography (Pond \& Vlachavas, 1983), relies on group-theoretical aspects of discrete lattices for determining the symmetries and the possible defect characteristics of interfaces (Sutton \& Balluffi, 1995).

One of the key concepts introduced in bicrystallography is the theory of the coincidence site lattice (CSL), which has played a major role in classifying experimentally observed interfaces in a wide array of inorganic polycrystalline material systems. The CSL corresponds to the 'intersection lattice' formed by overlapping two misoriented lattices (Grimmer, 1976). Associated with each CSL is the $\Sigma$ number, defined as the ratio of the volumes of the primitive unit cell of the CSL and the underlying single-crystal lattice. Therefore, $\Sigma$ quantifies the extent of overlap between the two misoriented lattices. An example of this is shown in Fig. 1(a) for a two-dimensional square lattice, where the two overlapping lattices are rotated by $\theta=2 \tan ^{-1}(1 / 3) \approx 36.87^{\circ}$ with respect to each other. The CSL obtained has an area five times the original lattice, and hence $\Sigma=5$.

From an experimental perspective, the algorithm for determining the $\Sigma$ value of an interface is straightforward. First, the lattice orientations of the adjoining grains are measured through orientation imaging microscopy either in the scanning (Schwartz et al., 2009) or the transmission mode (Trimby, 2012; Caswell et al., 2009). The misorientation matrix $M$ is then computed and $\Sigma$ is determined by the least positive integer such that $\Sigma M$ is an integral matrix. However, this algorithm is very sensitive to the precision with which the lattice orientations are measured. For example, shown in Fig. $1(b)$ is the bicrystal pattern obtained with a misorientation of $\theta=2 \tan ^{-1}(18 / 53)=37.52^{\circ}$. As is evident from this illustration, while the pattern changes ever so slightly from the $\Sigma=5$ bicrystal, the $\Sigma$ value associated with $\theta=37.52^{\circ}$ is 3133 . This large difference in $\Sigma$ stems from the fact that the CSL theory relies on the exact coincidence of lattice points. Therefore, a small error in the experimentally measured misorientation matrix can lead to a large deviation in the computed $\Sigma$ of the interface. This issue is particularly troublesome for interfaces in low-symmetry crystal systems where a priori enumeration of $\Sigma$ generating misorientations does not exist.

In this issue, Runnels presents a physically intuitive and mathematically elegant framework to overcome the challenges of the discrete nature of the CSL theory (Runnels, 2017). The central idea developed in this article is that $\Sigma$ can be computed by determining the extent of overlap between the atomic density fields of the two overlapping lattices. First, instead of representing atoms as a discrete set of points, they are expressed using continuous functions representing their probability densities. The functions used to represent the atomic density are called mollifiers (Friedrichs, 1944) and have unique properties that simplify the mathematics underlying the computation of the extent of overlap between two lattice fields. Bump functions such as those shown in Fig. 1 of 


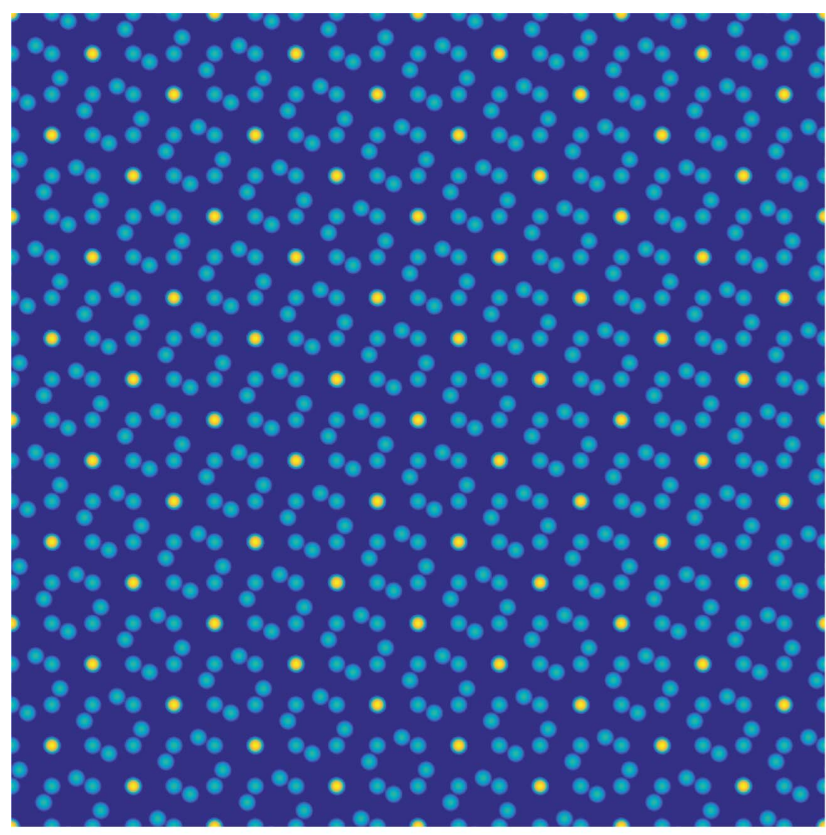

(a) $\Sigma=5 \quad \theta \approx 36.87^{\circ}$

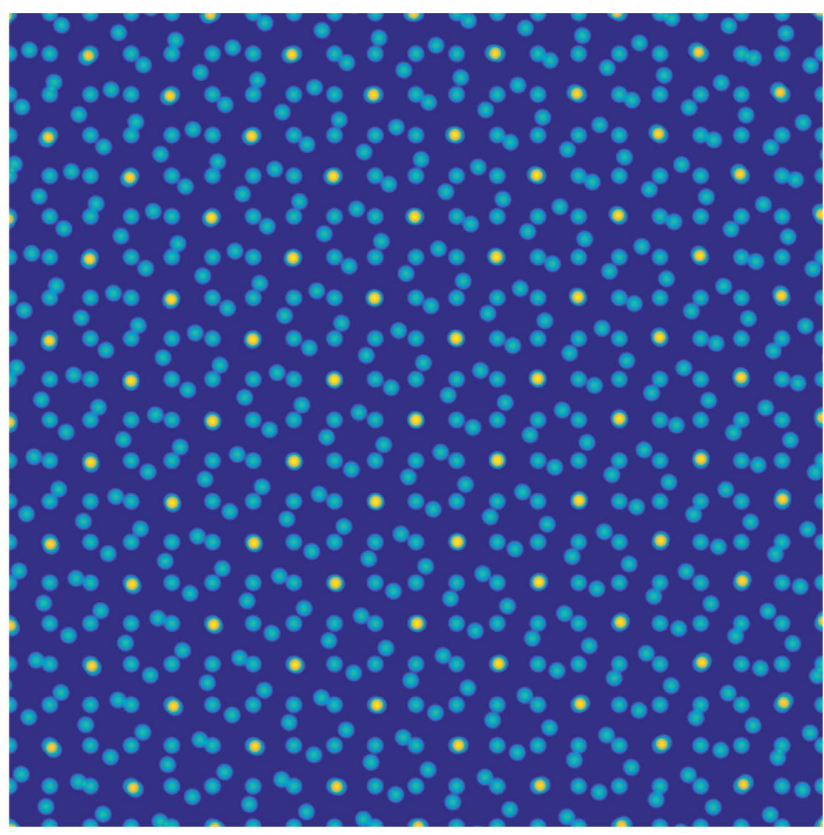

(b) $\Sigma=3133 \quad \theta \approx 37.52^{\circ}$

Figure 1

The irregular nature of the $\Sigma$ function is illustrated. In $(a)$, the bicrystal pattern obtained of a $\Sigma 5$ CSL, which corresponds to a misorientation of $\theta=36.87^{\circ}$, is shown. In $(b)$, a bicrystal pattern with misorientation $\theta=37.52^{\circ}$ is shown. While there is only a slight change in the extent of overlap between the lattices from $(a)$ to $(b)$, the value of $\Sigma$ changes from 5 to 3133. In fact, for all misorientations where $\tan \theta / 2$ is irrational, a coincidence site lattice does not exist and $\Sigma=\infty$. The atoms in this illustration are represented using the mollifier $\Phi=\left\{\exp \left[1 /\left(1-x^{2}\right)\right], x \in[-1,1], 0\right.$ else $\}($ Runnels, 2017).

Runnels (2017) are example of such mollifiers. Truncated Gaussian functions typically suffice as mollifier approximants and are convenient for analysis. Mollifiers, whose integral in space is unity, serve as intuitive representations of atomic density. For example, the width of the mollifiers (e.g. in a bump function) can be tuned using a parameter $\varepsilon$ that serves as a proxy for temperature.

Additionally, the extent of overlap between two lattices is shown to correspond to the integral of the product of the two lattice density fields that are misoriented with respect to each other. This integral is computed efficiently using a Fourier representation of the periodic lattice densities. By introducing a continuous functional representation of the atoms in the lattice sites, the discrete nature of the $\Sigma$ function is 'mollified'. That is, $\Sigma$ is now a continuously varying, smooth function of the lattice misorientation. The value of $\Sigma$ also changes with temperature as the parameter $\varepsilon$ in the mollifier depends on the temperature of the system. Therefore, this theory will facilitate an accurate determination of the $\Sigma$ associated with experimentally measured misorientations and can further help validate theoretically predicted CSL rotations in lowsymmetry material systems.

Finally, the lack of simple relationships between interfacial crystallography and properties has been identified as one of the key challenges in designing structural alloys for extreme environments. Therefore, it is desirable to utilize the quantities that represent the extent of overlap, given by $\Sigma$ for the three-dimensional lattices and by $\Gamma$ for the planar density of coincidence sites (Banadaki \& Patala, 2015), to predict properties such as interfacial energy. Unfortunately, the traditional definition of $\Sigma$ has not led to any useful or general criteria for correlating interfacial crystallography with properties. It is anticipated, however, that the regularized version of the $\Sigma$ function introduced by Runnels will provide a pathway for developing predictive rules for interfacial properties based on a simple geometrical framework. Such rules, elucidating the interfacial crystallography-property relationships, will help provide a key component in the microstructure-sensitive design of structural materials.

\section{References}

Banadaki, A. D. \& Patala, S. (2015). J. Appl. Cryst. 48, 585-588.

Caswell, T. A., Ercius, P., Tate, M. W., Ercan, A., Gruner, S. M. \& Muller, D. A. (2009). Ultramicroscopy, 109, 304-311.

Friedrichs, K. O. (1944). Trans. Am. Math. Soc. 55, 132-151.

Grimmer, H. (1976). Acta Cryst. A32, 783-785.

Pond, R. \& Vlachavas, D. (1983). Proc. R. Soc. London A, 386, 95143.

Runnels, B. (2017). Acta Cryst. A73, 87-92.

Schwartz, A. J., Kumar, M., Adams, B. L. \& Field, D. P. (2009). Electron Backscatter Diffraction in Materials Science, Vol. 2. Springer.

Sutton, A. P. \& Balluffi, R. W. (1995). Interfaces in Crystalline Materials, ch. 1, pp. 3-69. Oxford: Clarendon Press.

Trimby, P. W. (2012). Ultramicroscopy, 120, 16-24. 